\title{
Type D Personality and Heart Disease: Walking the Line Between Enthusiasm and Disbelief
}

\author{
Gesine Grande, Dr. p.h. • Matthias Romppel, MSc • \\ Jürgen Barth, PhD
}

Published online: 20 April 2012

(C) The Society of Behavioral Medicine 2012

\begin{abstract}
After many years of enthusiastic reports on the prognostic potential of the type $\mathrm{D}$ construct in patients with coronary heart disease, a critical discussion of methodological shortcomings of type D research was initiated recently. In their letter, de Voogd and colleagues summarize some of the critical issues we mentioned in our meta-analysis, issues that have been well documented elsewhere [2]. We agree with de Voogd and colleagues that small sample sizes, overfitted regression models, and the predominant use of dichotomized type D categories are methodological shortcomings of many primary studies on the effects of type $\mathrm{D}$ on prognosis in cardiac patients. That may lead to a higher probability for an overestimation of effects and spurious results, but it does not generate positive results per se.

We also agree that a meta-analysis cannot overcome methodological shortcomings of primary studies. Therefore, we conducted sensitivity analyses and carefully described potential weaknesses and strengths of the included primary studies. Our meta-analytic conclusion that there is a prognostic association of type $\mathrm{D}$ with mortality was qualified by
\end{abstract}

G. Grande $(\bowtie) \cdot$ M. Romppel

Faculty of Applied Social Sciences,

Leipzig University of Applied Sciences,

POB 301166,

04251 Leipzig, Germany

e-mail: grande@fas.htwk-leipzig.de

J. Barth

Institute of Social and Preventive Medicine (ISPM), Division of Social and Behavioral Health Research, University of Bern, Bern, Switzerland three other important points: (1) studies with lower methodological quality report have larger effects, (2) the effects decline considerably over time (as reported previously for other personality constructs such as the type A behavior pattern and also for biomedical research [1]), and (3) type $\mathrm{D}$ personality may not be associated with prognosis in chronic heart failure patients. Despite the overall significant meta-analytic risk estimate, these caveats raise doubt regarding the prognostic value of type D. Moreover, our work underscores the necessity and utility of quantitative reviews to identify problems in a body of literature.

The assertion by de Voogd et al. to banish the concept of type D personality seems premature based upon the available evidence. Aside from additional prospective studies, the most straightforward approach would be to reanalyze primary data from available type $\mathrm{D}$ studies with appropriate regression models and in an individual patient data metaanalysis to obtain greater statistical precision [2]. Such research incorporating narrative reviews and quantitative meta-analysis will provide the most defensible conclusion whether type $\mathrm{D}$ personality is associated with prognosis in CHD patients or not.

\section{References}

1. Ioannidis JP, Panagiotou OA. Comparison of effect sizes associated with biomarkers reported in highly cited individual articles and in subsequent meta-analyses. JAMA. 2011; 305: 2200-2210.

2. Smith TW. Toward a more systematic, cumulative, and applicable science of personality and health: Lessons from type D personality. Psychosom Med. 2011; 73: 528-532. 